\title{
A STRUCTURE THEOREM FOR THE POLARS OF UNITARILY INVARIANT NORMS
}

\author{
GOVIND S. MUDHOLKAR AND MARSHALL FREIMER
}

\begin{abstract}
The unitarily invariant norms of matrices, or operators, are essentially the symmetric norms of their singular values. A subclass of these norms depending upon only a few largest of the singular values is considered, and the polars of these norms are characterized. The result is then used to obtain generalizations of some well-known inequalities. The implications for operators on infinite-dimensional spaces are discussed.
\end{abstract}

1. Introduction and summary. The unitarily invariant (UI) norms were introduced by von Neumann [13] for the purpose of metrizing matrix spaces. He, together with his associates, established that the class of UI norms of $n \times n$ complex matrices coincides with the class of symmetric gauge functions (SGF's) of their singular values. These norms have now been variously generalized and utilized in several contexts. For example, Schatten $[\mathbf{1 0}, 11]$ defines norms on two-sided ideals of completely continuous operators on an arbitrary Hilbert space; Fan [2] obtains results relevant to these norms. The von Neumann norms play a crucial role in the study of function spaces and group representations (see, e.g., McCarthy [7] and Kunze [6]) and in obtaining certain bounds of importance in quantum field theory (see Simon [12]). More recently, Bhatia [1] has used the UI norms in the analysis of spectral variation. For historical perspectives and surveys of UI norms, see Schatten [10, 11], Hewitt and Ross [5], Gohberg and Krein [3] and Simon [12].

In his basic paper, von Neumann [13] studied various properties of SGF's and the corresponding UI norms. In particular, he showed that the polar of a UI norm is itself a UI norm. He illustrated the result using the usual $p$-norms. In this note we present a structure theorem for the polars of a class of UI norms. As an application of this result, we obtain the polars of Ky Fan's $k$-norms in explicit form and also an extension of Hölder's inequality. Some remarks on the spaces $c_{\phi}$, analogous to the operator spaces $c_{p}$ (see McCarthy [7]), related to these norms follow.

The basic facts relevant to the purpose of this paper are summarized in $\$ 2$. $\$ 3$ contains the main results. $\S 4$ is given to some applications and implications.

2. Preliminaries: SGF's and UI norms. Let $H$ be a Hilbert space of finite dimension $n$. Let $\mathrm{U}(H)$ be the group of all unitary operators in $\mathrm{B}(H)$, the linear space of all bounded operators on $H$. A norm $\|\cdot\|$ on $\mathrm{B}(H)$ is said to be UI, i.e.,

Received by the editors January 11, 1984 and, in revised form, October 2, 1984.

1980 Mathematics Subject Classification. Primary 15A60, 47A30; Secondary 15A39, 15A42, 46B10.

Kev words and phrases. Symmetric gauge functions, Hölder's inequality, conjugates, $c_{p}$. 
unitarily invariant, if for $A \in \mathrm{B}(H)$ and $U, V \in \mathrm{U}(H),\|A\|=\|U A V\|$. Construction of such norms requires SGF's, i.e., symmetric gauge functions on $R^{n}$.

Following von Neumann, a gauge function, i.e., a norm, $\phi: R^{n} \rightarrow R^{1}$ is said to be symmetric if (i) $\phi(\mathbf{x}) \geqslant 0$, with equality iff $\mathbf{x}=\mathbf{0}$, (ii) $\phi(c \mathbf{x})=|c| \phi(\mathbf{x})$ for any scalar $c$, and (iii) $\phi(\mathbf{x}+\mathbf{y}) \leqslant \phi(\mathbf{x})+\phi(\mathbf{y})$, for $\mathbf{x}=\left(x_{1}, x_{2}, \ldots, x_{n}\right) \in R^{n}, \mathbf{y} \in R^{n}$, and if it also satisfies

$$
\phi\left(\varepsilon_{1} x_{i_{1}}, \varepsilon_{2} x_{i_{2}}, \ldots, \varepsilon_{n} x_{i_{n}}\right)=\phi(\mathbf{x}),
$$

for any permutation $\left(i_{1}, i_{2}, \ldots, i_{n}\right)$ of $(1,2, \ldots, n)$ and any combination $\varepsilon_{i}= \pm 1$ of the signs.

Let $\Phi_{n}$ denote the class of all SGF's on $R^{n}$ as defined above. For any $\phi \in \Phi_{n}$ and $\mathbf{y} \in R^{n}$, define the dual

$$
\phi^{0}(\mathbf{y})=\max _{\mathbf{x}} \mathbf{x}^{\prime} \mathbf{y} / \phi(\mathbf{x})
$$

Alternatively, $\phi^{0}(\mathbf{y})$ is the maximum of $\mathbf{x}^{\prime} \mathbf{y}$ over the set $\{\mathbf{x} \mid \phi(\mathbf{x}) \leqslant 1\}$. Then $\phi^{0} \in \Phi_{n}$ and $\left(\phi^{0}\right)^{0}=\phi . \phi^{0}$ is known variously as the conjugate (von Neumann, Hewitt and Ross), the associate (Schatten) or the polar (Rockafellar [9]) of $\phi$.

Now for $A \in \mathrm{B}(H)$ let $\alpha_{1} \geqslant \alpha_{2} \geqslant \cdots \geqslant \alpha_{n} \geqslant 0$ be the singular values of $A$, i.e., the eigenvalues of $\left(A A^{*}\right)^{1 / 2}$. A major accomplishment of von Neumann's paper [13] is a demonstration of the fact that for any SGF $\phi \in \Phi_{n}$

$$
\|A\|_{\phi}=\phi\left(\alpha_{1}, \alpha_{2}, \ldots, \alpha_{n}\right)
$$

is a UI norm on $\mathrm{B}(H)$. Indeed every UI norm is generated in this manner by some SGF (see Schatten [10]). Furthermore,

$$
\|A\|=\sup _{B \neq 0} \frac{|\operatorname{tr} A B|}{\|B\|_{\phi}}
$$

is the UI norm generated by the polar $\phi^{0} \in \Phi_{n}$ of $\phi$ (see Hewitt and Ross [5, p. 703]). Call $\|A\|_{\phi^{0}}$, i.e., $\|A\|$ of (2.4), the polar of $\|A\|_{\phi}$.

3. The main result. For any $\mathbf{x}=\left(x_{1}, x_{2}, \ldots, x_{n}\right) \in R^{n}$, let $x_{(1)} \geqslant x_{(2)} \geqslant \cdots \geqslant 0$ denote the ordered values of $\left|x_{i}\right|, i=1,2, \ldots, n$. For any integer $m, m \leqslant n$, and $\phi \in \Phi_{m}$ let

$$
\phi_{m}(\mathbf{x})=\phi\left(x_{(1)}, x_{(2)}, \ldots, x_{(m)}\right) .
$$

Then it is easy to verify that $\phi_{m}$ defines an SGF on $R^{n}$. That is, $\phi_{m} \in \Phi_{n}$ and, in the notation of $\S 2$, the corresponding

$$
\|A\|_{\phi_{m}}=\phi\left(\alpha_{1}, \alpha_{2}, \ldots, \alpha_{m}\right)
$$

is a UI norm on $\mathrm{B}(H)$. In this section we characterize the polar $\phi_{m}^{0}$ of $\phi_{m} \in \Phi_{n}$ in terms of the polar $\phi^{0}$ of $\phi \in \Phi_{m}$ and consequently obtain the polar of $\|A\|_{\phi_{m}}$. In order to describe and prove the main results we need the following two lemmas. 
LEMMA 3.1. If $b_{1} \geqslant b_{2} \geqslant \cdots \geqslant b_{n} \geqslant 0$ and $m$ is an integer $1 \leqslant m \leqslant n$, then there exists a unique integer $k, 0 \leqslant k<m$, such that

$$
b_{k+1} \leqslant \sum_{j=k+1}^{n} b_{j} /(m-k)<b_{k},
$$

the second inequality being inoperative if $k=0$.

Proof. For $r=1,2, \ldots, m$, let

$$
\beta_{r}=(m-r) b_{r}-\sum_{j=r+1}^{n} b_{j} .
$$

Then

$$
\beta_{r}-\beta_{r+1}=(m-r)\left(b_{r}-b_{r+1}\right) \geqslant 0,
$$

for $r=1,2, \ldots, m-1$, and $\beta_{m}=-\sum_{m+1}^{n} b_{j} \leqslant 0$. Hence, there is a unique $k$ such that $\beta_{k}>0 \geqslant \beta_{k+1}$, i.e. the $k$ which satisfies (3.3).

LEMMA 3.2. Let $b_{1} \geqslant b_{2} \geqslant \cdots \geqslant b_{s} \geqslant 0$, and $\phi \in \Phi_{s}$. Then the maximum value $\phi^{0}(\mathbf{b})$ of the sum $\sum_{1}^{s} a_{i} b_{i}$ subject to $\phi(\mathbf{a})=\phi\left(a_{1}, a_{2}, \ldots, a_{s}\right) \leqslant 1$ is attained at a vector which satisfies $a_{1} \geqslant a_{2} \geqslant \cdots \geqslant a_{s} \geqslant 0$.

Proof. The maximum must occur when the coordinates of $\mathbf{a}$ and $\mathbf{b}$ are similarly ordered. Otherwise by the rearrangement theorem, e.g., see Hardy, Littlewood and Pólya [4], the sum $\sum_{1}^{s} a_{i} b_{i}$ could be increased without violating the restriction $\phi(\mathbf{a}) \leqslant 1$.

THEOREM 3.3. Let $\phi_{m} \in \Phi_{n}$ be the $S G F$ derived from $\phi \in \Phi_{m}, m \leqslant n$, according to (3.1). Then the polar $\phi_{m}^{0} \in \Phi_{n}$ of $\phi_{m}$ is given by

$$
\phi_{m}^{0}(\mathbf{y})=\phi^{0}\left(y_{(1)}, y_{(2)}, \ldots, y_{(k)}, \bar{y}, \bar{y}, \ldots, \bar{y}\right),
$$

where $\phi^{0} \in \Phi_{m}$ is the polar of $\phi, y_{(1)} \geqslant y_{(2)} \geqslant \cdots \geqslant y_{(n)} \geqslant 0$ are the ordered values of the magnitudes $\left|y_{i}\right|$ of the coordinates of $\mathbf{y} \in R^{n}, k$ is obtained by applying Lemma 3.1 to the $y_{(i)}$ 's, and $\bar{y}=\sum_{k+1}^{n} y_{(j)} /(m-k)$.

Proof. Since we are working with SGF's, assume with no loss of generality that $y_{1} \geqslant y_{2} \geqslant \cdots \geqslant y_{n} \geqslant 0$. By definition, we have

$$
\phi_{m}^{0}(\mathbf{y})=\max \sum_{1}^{n} x_{i} y_{i},
$$

where the maximum is over the set $\left\{\mathbf{x} \mid \phi_{m}(\mathbf{x})=\phi\left(x_{(1)}, x_{(2)}, \ldots, x_{(m)}\right) \leqslant 1\right\}$, and the $x_{(i)}$ 's are the ordered values of $\left|x_{i}\right|, i=1,2, \ldots, n$. But

$$
\sum_{1}^{n} x_{i} y_{i} \leqslant \sum_{1}^{m-1} x_{(i)} y_{i}+x_{(m)} \sum_{m}^{n} y_{i},
$$

with equality if, and only if, $x_{1} \geqslant x_{2} \geqslant \cdots \geqslant x_{m}=x_{m+1}=\cdots=x_{n} \geqslant 0$. Hence,

$$
\phi_{m}^{0}(\mathbf{y})=\max _{S_{0}}\left\{\sum_{1}^{m-1} x_{i} y_{i}+x_{m} y_{[m]}\right\},
$$

where $y_{[m]}$ denotes the tail-sum $\sum_{m}^{n} y_{i}$, and

$$
S_{0}=\left\{\mathbf{x} \in R^{m} \mid x_{1} \geqslant x_{2} \geqslant \cdots \geqslant x_{m} \geqslant 0, \phi\left(x_{1}, x_{2}, \ldots, x_{m}\right) \leqslant 1\right\} .
$$


Now suppose that the maximum in (3.8) is known to occur for $\mathbf{x}$ in the subset of $S_{0}$ defined by

$$
S_{j}=\left\{\mathbf{x} \in S_{0} \mid x_{m-j}=x_{m-j+1}=\cdots=x_{m}\right\} .
$$

For $j=0$ this obviously is so. We will now prove that if the maximum is in $S_{j}$ and $j<m-k-1$, then in fact the maximum is in $S_{j+1}$. This will permit us to conclude that the maximum in (3.8) is in $S_{m-k-1}$.

To prove the inductive step, for any $\mathbf{x} \in S_{j}$ define $\mathbf{x}^{*}$ as follows:

$$
x_{i}^{*}= \begin{cases}x_{i}, & i=1,2, \ldots, m-j-2, \\ \frac{x_{m-j-1}+(j+1) x_{m-j}}{j+2}, & i=m-j-1, \ldots, m .\end{cases}
$$

Then by the symmetry and convexity of $\phi$,

$$
\phi\left(x_{1}^{*}, \ldots, x_{m}^{*}\right) \leqslant \phi\left(x_{1}, \ldots, x_{m}\right) \leqslant 1,
$$

so that $\mathbf{x}^{*} \in S_{j+1}$. In order to show that

$$
\sum_{1}^{m-1} x_{i} y_{i}+x_{m} y_{[m]} \leqslant \sum_{1}^{m-1} x_{i}^{*} y_{i}+x_{m}^{*} y_{[m]}
$$

we substitute the definition of $x_{i}^{*}$. Then the terms with $i \leqslant m-j-2$ cancel, and it remains to show that

$$
x_{m-j-1} y_{m-j-1}+x_{m-j} \sum_{m-j}^{n} y_{i} \leqslant \frac{x_{m-j-1}+(j+1) x_{m-j}}{j+2} \sum_{m-j-1}^{n} y_{i} .
$$

This is equivalent to showing that

$$
0 \leqslant\left(x_{m-j-1}-x_{m-j}\right)\left(\sum_{m-j}^{n} y_{i}-(j+1) y_{m-j-1}\right) .
$$

The first factor in (3.15) is nonnegative since $\mathbf{x} \in S_{0}$. In the notation of the proof of Lemma 3.1, as applied to the $y$ 's, the second factor is $-\beta_{m-j-1}$. This is nonnegative as long as $j<m-k-1$.

We have just proved that

$$
\phi_{m}^{0}(\mathbf{y})=\max _{\mathbf{x} \in S_{m-k-1}}\left\{\sum_{1}^{m-1} x_{i} y_{i}+x_{m} y_{[m]}\right\} .
$$

Since $x_{k+1}=x_{k+2}=\cdots=x_{m}$, this is equal to

$$
\max _{\mathbf{x} \in S_{m-k-1}}\left\{\sum_{1}^{k} x_{i} y_{i}+\sum_{k+1}^{m} x_{i} \bar{y}\right\}, \quad \text { where } \bar{y}=\frac{1}{m-k} \sum_{k+1}^{n} y_{i} .
$$

Because the coefficients of $x_{k+1}, \ldots, x_{m}$ are equal, this in turn equals

$$
\max _{\mathbf{x} \in S_{0}}\left\{\sum_{1}^{k} x_{i} y_{i}+\sum_{k+1}^{m} x_{i} \bar{y}\right\} \text {. }
$$

But $k$ has been chosen to make $y_{1} \geqslant \cdots \geqslant y_{k} \geqslant \bar{y}$. Hence we can apply Lemma 3.2 
to see that the last expression equals

$$
\max _{\phi(x) \leqslant 1}\left\{\sum_{1}^{k} x_{i} y_{i}+\sum_{k+1}^{m} x_{i} \bar{y}\right\}=\phi^{0}\left(y_{1}, y_{2}, \ldots, y_{k}, \bar{y}, \ldots, \bar{y}\right) .
$$

This completes the proof of the theorem.

Combining the definitions of a UI norm $\|A\|_{\phi}$ at (2.3), its polar $\|A\|_{\phi^{0}}$ at (2.4), and of a trimmed SGF $\phi_{m}$ at (3.1), we get

COROllaRY 3.4. For any $\phi \in \Phi_{m}, m \leqslant n$, the trimmed $S G F \phi_{m}$ defines a UI norm on $\mathrm{B}(H)$. Its polar is the UI norm defined by $\phi_{m}^{0}$ at (3.5).

4. Applications and implications. The following observations outline some possible uses, further consequences and suggestions resulting from the main structural result presented in the previous section.

(1) Two classes of SGF's commonly occur in literature as illustrations. For $1 \leqslant p \leqslant \infty$ and $\mathbf{x} \in R^{n}, \phi(\mathbf{x})=\left(\sum_{1}^{n}\left|\mathbf{x}_{i}\right|^{p}\right)^{1 / p}$ is an SGF in $\Phi_{n}$ with $\phi^{0}(\mathbf{x})=$ $\left(\sum_{1}^{n}\left|x_{i}\right|^{q}\right)^{1 / q}, q^{-1}=1-p^{-1}$. The UI norms of the operators generated by these SGF's are known as the Schatten $p$-norms. If $m \leqslant n$ is an integer, $\mathbf{x} \in R^{n}$, then in the notation of $\S 3, \phi(\mathbf{x})=\sum_{1}^{m} x_{(i)}$ is an SGF, the corresponding UI norm $\|A\|_{\phi}$ being known as a Ky Fan $k$-norm. The results in $\$ 3$ provide the duals of these norms.

(2) If $a_{1} \geqslant a_{2} \geqslant \cdots \geqslant a_{n} \geqslant 0$ and $b_{1} \geqslant b_{2} \geqslant \cdots \geqslant b_{n} \geqslant 0, m \leqslant n$ and $p \geqslant 1$, then taking $\phi\left(a_{1}, a_{2}, \ldots, a_{m}\right)=\left(\sum_{1}^{m} a_{i}^{p}\right)^{1 / p}$ we get

$$
\sum_{1}^{n} a_{i} b_{i} \leqslant\left(\sum_{1}^{m} a_{i}^{p}\right)^{1 / p}\left(\sum_{1}^{k} b_{i}^{q}+(m-k) \bar{b}^{q}\right)^{1 / q},
$$

where $q^{-1}=1-p^{-1}$, and $k$ is as in $\S 3$. This generalization of Hölder's inequality is proved by Mudholkar, Freimer and Subbaiah [8] using arguments of convex analysis.

(3) If $0<a_{1} \leqslant a_{2} \leqslant \cdots \leqslant a_{n}$, and $p<1$, then $\left(\sum_{1}^{n} a_{i}^{p}\right)^{1 / p}$ is a concave homogeneous function of $\left(a_{1}, a_{2}, \ldots, a_{n}\right)$. If $b_{1} \geqslant b_{2} \geqslant \cdots \geqslant b_{n} \geqslant 0, m \leqslant n$, and $q^{-1}=1$ $-p^{-1}$, then Mudholkar, Freimer and Subbaiah [8] also established the following reversal of Hölder's inequality:

$$
\sum_{1}^{n} a_{i} b_{i} \geqslant\left(\sum_{1}^{m} a_{i}^{p}\right)^{1 / p}\left(\sum_{1}^{k} b_{i}^{q}+(m-k) \bar{b}^{q}\right)^{1 / q} .
$$

The analogous reversal of the more general inequality in $\$ 3$ can be developed in a straightforward manner.

(4) If $f$ is a real valued convex function on $R^{n}$, then

$$
f^{c}(\mathbf{y})=\sup _{\mathbf{x} \in R^{n}}\left[\mathbf{y}^{\prime} \mathbf{x}-f(\mathbf{x})\right],
$$

the Fénchél conjugate, leads to the inequality $\mathbf{y}^{\prime} \mathbf{x} \leqslant f(\mathbf{x})+f^{c}(\mathbf{y})$. Analogues of the results in $\$ 3$ for Fénchél conjugates exist and consequent matrix inequalities follow.

(5) A substantial implication of the structural result pertains to the problem of metrizing the completely continuous operators on an infinite-dimensional Hilbert space (see Schatten [11] and Simon [12]). 
If $A$ is a completely continuous operator on a Hilbert space of countable dimension, then the singular values of $A$ (including multiplicities) can be written as a nonincreasing sequence $\left\{\alpha_{i}(A)\right\}$ of nonnegative real numbers which converges to zero. If $U$ and $V$ are unitary operators, then $\alpha_{i}(U A V)=\alpha_{i}(A)$ for all $i$. Conversely, if $B$ is also completely continuous and $\alpha_{i}(B)=\alpha_{i}(A)$ for all $i$, then $B=U A V$ with $U$ and $V$ unitary.

If $1 \leqslant p<\infty$, we can define

$$
\|A\|_{p}=\lim _{n \rightarrow \infty}\left[\sum_{i=1}^{n} \alpha_{i}(A)^{p}\right]^{1 / p} .
$$

Those completely continuous operators for which this is finite form a Banach space $c_{p}$ with this as norm (see McCarthy [7]). If $p>1$ the space is reflexive, and $\left(c_{p}\right)^{*}=c_{q}$, where $p^{-1}+q^{-1}=1$. However, $c_{1}$ is not reflexive, and $\left(c_{1}\right)^{*}$ consists of all bounded operators.

More generally, let $\phi$ be any symmetric gauge function defined on the space of sequences having only a finite number of nonzero terms. Then we can still use definition (2.2) to define the polar $\phi^{0}$. (Furthermore, if $\phi_{m}$ is defined by (3.1) then $\phi_{m}^{0}$ is still given by Theorem 3.3.) Both $\phi$ and $\phi^{0}$ can be extended to the space of sequences converging to zero by taking limits. Of course, for some such sequences, $\phi$ or $\phi^{0}$ may be equal to $+\infty$.

The unitarily invariant norms for completely continuous operators are of the form $\|A\|_{\phi}=\phi\left(\left\{\alpha_{i}(A)\right\}\right)$. Those $A$ for which $\|A\|_{\phi}<\infty$ form a Banach space $c_{\phi}$. We still have the equation

$$
\|A\|_{\phi^{0}}=\sup _{B \neq 0} \frac{|\operatorname{tr} A B|}{\|B\|_{\phi}} .
$$

This leads to the inclusion relation

$$
\left(c_{\phi}\right)^{*} \supset c_{\phi^{0}}
$$

with equality holding if $c_{\phi}$ is reflexive. It so happens that $c_{\phi_{m}}$ is not reflexive but $\left(c_{\phi_{m}}\right)^{*}=c_{\phi_{m}^{0}}$ still holds.

\section{REFERENCES}

1. R. Bhatia, Analysis of spectral variation and some inequalities, Trans. Amer. Math. Soc. 272 (1982), 323-331.

2. K. Fan, Maximum properties and inequalities for the eigenvalues of completely continuous operators, Proc. Nat. Acad. Sci. U.S.A. 37 (1951), 760-766.

3. I. C. Gohberg and M. G. Krein, Introduction to the theory of linear non-self adjoint operators, Amer. Math. Soc. Transl. 18 (1969).

4. G. H. Hardy, J. E. Littlewood and G. Polya, Inequalities, Cambridge Univ. Press, 1934.

5. E. Hewitt and K. A. Ross, Abstract harmonic analysis, Vol. II, Springer-Verlag, Berlin, 1970.

6. R. Kunze, $L^{p}$-Fourier transforms on locally compact unimodular groups, Trans. Amer. Math. Soc. 89 (1958), 519-540.

7. C. A. McCarthy, $c_{p}$, Israel J. Math. 5 (1967), 249-271.

8. G. S. Mudholkar, M. Freimer and P. Subbaiah, An extension of Hölder's inequality, J. Math. Anal. Appl. 102 (1984), 435-441. 
9. R. T. Rockafellar, Convex analysis, Princeton Univ. Press, Princeton, N.J., 1970.

10. R. Schatten, $A$ theory of cross-spaces, Ann. of Math. Studies, No. 26, Princeton Univ. Press, Princeton, N.J., 1950.

11. N_ Norm ideals of completely continuous operators, Springer-Verlag, Berlin, 1960.

12. B. Simon, Trace ideals and their applications, Cambridge Univ. Press, 1979.

13. J. von Neumann. Some matrix inequalities and metrization of matric spaces, Tomsk. Univ. Rev. 1 (1937), 286-300 (also Collected Works, Vol. IV).

Department of Statistics and Graduate School of Management, University of Rochester, ROCHESTER, NEW YORK 14627 